CERN-TH/97-361

hep-ph/9712343

November 1997

\title{
The Role of Scalar and Pseudoscalar Fields in Determining Nucleosynthesis Bounds on the Scale of Supersymmetry Breaking
}

\author{
Tony Gherghetta* \\ Theory Division, CERN, CH-1211 Geneve 23, Switzerland
}

\begin{abstract}
The effect of spin- 0 goldstino superpartners is considered on the nucleosynthesis bounds arising when a superlight gravitino appears as an effective massless neutrino species. When the scalar and pseudoscalar superpartners are relativistic they will decouple at much later times than the goldstino and consequently will be the dominant effect when obtaining a nucleosynthesis bound on the scale of supersymmetry breaking. Assuming that the scalar and pseudoscalar fields decouple at a temperature no later than $\mathcal{O}(100) \mathrm{MeV}$, then typically the scale of supersymmetry breaking $\sqrt{F} \gtrsim 60 \mathrm{TeV}$. This corresponds to a lower bound on the gravitino mass $m_{3 / 2} \gtrsim 1 \mathrm{eV}$.
\end{abstract}

*Tony.Gherghetta@cern.ch 


\section{INTRODUCTION}

If supersymmetry is realised in nature then it is presumably broken at some energy scale and subsequently transmitted indirectly to the low energy observable mass spectrum [1]. While naturalness arguments essentially constrain the amount of supersymmetry breaking in the low energy mass spectrum, the scale at which supersymmetry is broken remains largely undetermined. In general, realistic supersymmetry breaking scenarios can be parametrised by a spurion chiral supermultiplet $(\phi, \psi, F)$ in which a nonzero vacuum expectation value for the F-term gives rise to the spontaneous breaking of supersymmetry at the scale $\sqrt{F}$. The fermion, $\psi$ represents the massless Nambu-Goldstone fermion which becomes part of the massive gravitino after the superHiggs effect. This fermion is referred to as the goldstino $\tilde{G}$. The gravitino mass $m_{3 / 2}$ is determined purely by gravitational interactions and is fixed by the

relation $F=\sqrt{3} m_{3 / 2} M_{P}$, where the reduced Planck mass $M_{P}=\sqrt{8 \pi G_{N}}=2.4 \times 10^{18} \mathrm{GeV}$ is related to the gravitational Newton constant $G_{N}$. When the gravitino mass $m_{3 / 2} \rightarrow 0$, the goldstino becomes the dominant component of the gravitino during interactions.

The mass of the spin-0 superpartner $\phi$, is however not fixed and can in general depend on nonminimal terms in the Kahler potential as well as terms in the superpotential. When the complex scalar is decomposed into real fields $\phi=(S+i P) / \sqrt{2}$, we need to consider the scalar mass $m_{S}$ and pseudoscalar mass $m_{P}$ as additional parameters to the supersymmetry breaking scale $F$. In this framework we will be interested in placing a lower bound on the scale of supersymmetry breaking from the effect of a superlight gravitino together with its scalar superpartners contributing as extra effective neutrino species during the nucleosynthesis epoch.

The effect of a superlight gravitino in the early universe was considered in a previous calculation [2]. There it was assumed that the spin-0 superpartners were essentially massless $\left(m_{S}, m_{P} \ll T\right.$, where $T$ is the temperature during the nucleosynthesis epoch) and that they decouple before the superlight gravitino. More recently, there have appeared additional papers [3 5] in which the general question of determining an effective gravitino Lagrangian have been addressed. In addition these references investigated the case of massive superpartner masses $m_{S}, m_{P} \gg T$ and noticed qualitatively different behaviour for scattering cross sections than the superlight scenario. However they did not address the question of superlight scalar superpartners during nucleosynthesis.

In this work we will consider the effect of the scalar superpartners in determining the nucleosynthesis bounds on the supersymmetry breaking scale $F$ and in particular consider the case of superlight masses for the scalars. It will be shown that $S$ and $P$ decouple much later than the goldstinos, so that the scalar and pseudoscalar contributions actually strengthen the bound considered in [2], when $m_{S}$ and $m_{P}$ are superlight as well. While we provide a more complete study of the effect of the scalar and pseudoscalar fields during nucleosynthesis, the result for superlight $S, P$ masses will agree with [6]. In section 2 we will consider the scattering cross sections for $S, P$ and $\tilde{G}$ which are relevant during the nucleosynthesis epoch. This will then allow us, in section 3, to obtain an expression for the corresponding decoupling temperature. We will see that this leads to a bound on the supersymmetry breaking scale, $F$ and consequently a lower bound on the gravitino mass $m_{3 / 2}$. The conclusion and final comments appear in section 4 . 


\section{SCATTERING CROSS SECTIONS}

We will assume that all gravitino interactions are dominated by a superlight goldstino in determining the gravitino scattering cross sections. The associated scattering cross sections of the scalar superpartners will also be considered when they are superlight as well. We will make the usual assumption that a massless and weakly interacting particle decouples when the relic abundance is frozen. Thus it will be sufficient to consider processes that change particle number. The scattering cross sections can be obtained once all the effective interactions between the relevant particle species is known. While the full supergravity formalism was employed in [7, [], it will be simpler instead to use the effective Lagrangian approach adopted by [3] where the effects of gravity are formally decoupled in the limit $M_{P} \rightarrow \infty$ while keeping $F$ fixed 1 . During the nucleosynthesis era the particle species that are assumed to be in thermal equilibrium besides $S, P$ and $\tilde{G}$ will be the photon $(\gamma)$, three families of massless neutrinos $\left(\nu_{i}\right)$ and fermions, including at least electrons $\left(e^{ \pm}\right)$and possibly muons $\left(\mu^{ \pm}\right)$. The temperature of the universe at these times will be in the range $\mathcal{O}(1-100) \mathrm{MeV}$.

\section{A. Goldstino annihilation}

The cross section for goldstino annihilation in the massless $S, P$ limit was previously calculated for various channels in [2,:7]. For goldstino annihilation into photons the result is

$$
\sigma(\tilde{G} \tilde{G} \rightarrow \gamma \gamma)=\frac{1}{64 \pi} \frac{\tilde{M}^{2}}{F^{4}} s^{2}
$$

where $s$ denotes the Mandelstam variable for the total energy in the center of mass frame and $\tilde{M}$ generically denotes the gaugino mass (the effects of neutralino mixing will be ignored in this paper). The result (11) is valid in the limit $m_{3 / 2}, m_{S}, m_{P} \ll \sqrt{s} \ll \tilde{M}$. It is also straightforward to obtain the annihilation cross section in the limit $\sqrt{s} \ll m_{S}, m_{P}, \tilde{M}$ for which one finds

$$
\sigma(\tilde{G} \tilde{G} \rightarrow \gamma \gamma)=\frac{1}{640 \pi} \frac{s^{3}}{F^{4}}
$$

This expression for the annihilation cross section agrees with the result derived by [3,5], where it was noted that the cancellations of the leading order terms change the massless $S, P$ limit cross section (1). These cancellations are obviously absent when one derives the cross section in the massless $S, P$ limit and there is no inconsistency with the previous literature [7, 8, 2], in which the massless limit was always the underlying assumption.

Similarly, the Goldstino annihilation into fermions was calculated in [2, 14]. As shown in [4] the cross section for annihilation into fermions is ambiguous up to an unknown parameter

\footnotetext{
${ }^{1}$ An alternative approach using a nonlinear realisation of the supersymmetry algebra can also be used [5].
} 
which represents the neglect of higher derivative terms in the effective Lagrangian approach. While this ambiguity will be seen to have no direct consequences on the results obtained in this paper, we will nevertheless use a minimum value of the cross section, namely [9]

$$
\sigma(\tilde{G} \tilde{G} \rightarrow f \bar{f})=\frac{1}{1280 \pi} \frac{s^{3}}{F^{4}}
$$

in order to derive a model independent bound.

The above annihilation channels into photons and fermions are not the only possible channels. Since the goldstino superpartners $S$ and $P$ are also assumed to be in thermal equilibrium we need to also consider the annihilation channels $\tilde{G} \tilde{G} \rightarrow S S, P P, S P$. The effective Langrangian governing these interactions is given by

$$
\mathcal{L}=-\frac{1}{2 \sqrt{2}} \frac{m_{S}^{2}}{F} S \tilde{G} \tilde{G}-\frac{i}{2 \sqrt{2}} \frac{m_{P}^{2}}{F} P \tilde{G} \tilde{G}+\text { h.c. }
$$

This leads to the following Goldstino annihilation cross sections into the scalar superpartners $S, P$ in the limit $m_{S}, m_{P} \ll \sqrt{s}$

$$
\begin{aligned}
\sigma(\tilde{G} \tilde{G} \rightarrow S S) & =\frac{1}{128 \pi} \frac{m_{S}^{8}}{F^{4}} \frac{1}{s}\left[\log \left(\frac{s}{m_{3 / 2}^{2}}\right)-2\right], \\
\sigma(\tilde{G} \tilde{G} \rightarrow P P) & =\frac{1}{128 \pi} \frac{m_{P}^{8}}{F^{4}} \frac{1}{s}\left[\log \left(\frac{s}{m_{3 / 2}^{2}}\right)-2\right], \\
\sigma(\tilde{G} \tilde{G} \rightarrow S P) & =\frac{1}{16 \pi} \frac{m_{S}^{4} m_{P}^{4}}{F^{4}} \frac{1}{s}\left[\log \left(\frac{s}{m_{3 / 2}^{2}}\right)-2\right] .
\end{aligned}
$$

Clearly in the massless limit these cross sections are significantly smaller than (1) and will contribute negligibly to the overall goldstino annihilation rate.

\section{B. $S$ and $P$ annihilation}

The annihilation channels available for $S$ and $P$ are similar to those for the goldstino, except that unlike goldstinos there are no fermion couplings in the leading order $1 / F$. Thus the effective Lagrangian describing their interactions will be the goldstino Lagrangian (田) together with the interaction with photons [3]

$$
\begin{aligned}
\mathcal{L}= & \frac{-1}{2 \sqrt{2}} \operatorname{Re}\left(\frac{\tilde{M}}{F}\right) S F_{\mu \nu} F^{\mu \nu}+\frac{1}{4 \sqrt{2}} \operatorname{Im}\left(\frac{\tilde{M}}{F}\right) S \epsilon^{\mu \nu \rho \sigma} F_{\mu \nu} F_{\rho \sigma} \\
& +\frac{1}{2 \sqrt{2}} \operatorname{Im}\left(\frac{\tilde{M}}{F}\right) P F_{\mu \nu} F^{\mu \nu}+\frac{1}{4 \sqrt{2}} \operatorname{Re}\left(\frac{\tilde{M}}{F}\right) P \epsilon^{\mu \nu \rho \sigma} F_{\mu \nu} F_{\rho \sigma}
\end{aligned}
$$

In the limit that $m_{S}, m_{P} \ll \sqrt{s}$ this leads to an annihilation cross section

$$
\sigma(S S \rightarrow \gamma \gamma)=\sigma(P P \rightarrow \gamma \gamma)=\frac{5}{64 \pi} \frac{|\tilde{M}|^{4}}{F^{4}} s
$$


obtained via $\mathrm{t}$ and $\mathrm{u}$-channel photon exchange. This cross section agrees with the result obtained by employing the full supergravity formalism []. Notice that the dependence on the Mandelstam variable $s$ is qualitatively different from that of (11) and we can already see that $S$ and $P$ interact more strongly with the thermal plasma when $T \lesssim \tilde{M}$. This will ultimately correspond to $S$ and $P$ decoupling later than the goldstino.

There are also possible annihilation channels into goldstino pairs due to the interactions (目). These are the inverse of the processes considered in (567) and give rise to similar cross sections for annihilation into goldstino pairs, namely $\sigma(S S \rightarrow \tilde{G} \tilde{G})=4 \sigma(\tilde{G} \tilde{G} \rightarrow$ $S S$ ) and similarly for $P$. The overall factor of 4 comes from the averaging over initial polarisations of the goldstino in the expressions for the unpolarised cross section (5-7). Again this annihilation channel will have a negligible effect on determining the decoupling temperature. Other channels such as $S \tilde{G} \rightarrow P \tilde{G}$ will also not be important.

The form of the $S, P$ couplings to photons (89) also allows for the channel $S f \rightarrow \gamma f$. A photon is exchanged in the t-channel and couples to fermion pairs in the standard way. In the limit $\sqrt{s} \gg m_{S}, m_{f}$ where $m_{f}$ is the fermion mass one finds that

$$
\sigma(S f \rightarrow \gamma f)=\frac{\alpha}{2} \frac{|\tilde{M}|^{2}}{F^{2}}\left[\log \left(\frac{s^{3}}{m_{S}^{4} m_{f}^{2}}\right)-\frac{7}{4}\right],
$$

where $\alpha$ is the electromagnetic fine structure constant and $f$ is a Dirac fermion (similarly for $P$ with the substitution $m_{S} \rightarrow m_{P}$ ). Notice that the dependence on the center of mass energy $\sqrt{s}$ is now logarithmic and no longer a power law like previous annihilation channels. This channel (known as the Primakoff reaction) was also considered in [6] but in the limit $m_{f} \gg E_{\gamma} \gg E_{S}$ and is also important for establishing bounds on the gravitino mass from supernova cooling [10,6]. When $m_{S} \rightarrow 0$ the cross section becomes singular due to collinear singularities. In order to regulate this infrared divergence we can introduce a thermal mass $M_{\gamma}$ for the photon in the background plasma. Without going into a full finite temperature calculation this will be a good approximation to leading log order [11]. Thus, in the massless $S$ limit the cross section (11) becomes

$$
\sigma(S f \rightarrow \gamma f)=\frac{\alpha}{2} \frac{|\tilde{M}|^{2}}{F^{2}}\left[\log \left(\frac{s}{M_{\gamma}^{2}}\right)-\frac{7}{4}\right],
$$

and similarly for $P$. This cross section will become important in obtaining the decoupling temperature of the $S, P$ superpartners.

Similarly $S$ and $P$ can both scatter off the background photons via a t and s-channel photon exchange with various particles in the final state. The scattering cross section for a photon in the final state in the massless $S, P$ limit is

$$
\sigma(S \gamma \rightarrow \gamma P)=\sigma(P \gamma \rightarrow \gamma S)=\frac{11}{192 \pi} \frac{|\tilde{M}|^{4}}{F^{4}} s .
$$

Similarly the cross section for pair production is

$$
\sigma(S \gamma \rightarrow f \bar{f})=\sigma(P \gamma \rightarrow f \bar{f})=\frac{\alpha}{6} \frac{|\tilde{M}|^{2}}{F^{2}}\left(1-\frac{4 m_{f}^{2}}{s}\right)^{3 / 2} .
$$


The process (13) is comparable to (10), while (14) is subdominant compared to (12) and neither will have a large effect in the determination of the decoupling temperature.

Up to now we have implicitly neglected the possibility of cubic scalar couplings in the previous goldstino and scalar channels. In general these cubic scalar interactions arise from nonminimal terms in the Kahler potential and superpotential terms [3]. While in all realistic models the cubic scalar couplings vanish it is interesting to comment on their effect if the scalar annihilation channel were to include such a term. For example, the effect of the scalar cubic couplings on the cross section (10) can be seen if we parametrise the interaction as $\mathcal{L}=-A_{S} / 3$ ! $S^{3}$. Including this term allows for an s-channel $S$ exchange diagram contributing to the annihilation into photons. In the massless $S$ limit we obtain

$$
\sigma(S S \rightarrow \gamma \gamma)=\frac{1}{64 \pi} \frac{|\tilde{M}|^{2}}{F^{2}}\left(5 \frac{|\tilde{M}|^{2}}{F^{2}} s+4 \sqrt{2} \frac{\operatorname{Re} \tilde{M}}{F} A_{S}+2 \frac{\left|A_{S}\right|^{2}}{s}\right)
$$

The interesting feature of the above cross section is that while at high energies we reproduce the result (10), at low energies the cross section may grow due to the $1 / s$ behaviour. Cosmologically this would mean that the scalar $S$ would actually come back into thermal equilibrium at late times if the coupling $A_{S}$ were large enough. Similarly a cubic scalar coupling of the form $\sim S^{2} P$ would modify (13) to a form like (15) via a u-channel scalar exchange diagram.

There are also dimension-6 terms $\sim S S F_{\mu \nu} F^{\mu \nu}$ (similarly $S P, P P$ ) which in a more general framework must also be included'? The coupling is controlled by the second derivative of the gauge kinetic function. Again for simplicity we will assume this term to be absent.

\section{THE DECOUPLING TEMPERATURE}

The decoupling temperature of a particle species in the early universe is obtained in general by solving a set of coupled Boltzmann equations. However, in practice a reasonable estimate of the decoupling temperature can be obtained by checking when the interaction rate of a particular particle species begins to fall behind the Hubble expansion rate $H$. The interaction rate for the scattering process $1+2 \rightarrow \mathcal{F}$ into a set of final states $\mathcal{F}$ is related to the thermally averaged cross section times velocity, which can be calculated using the definition [12]

$$
\left\langle\sigma v_{M \varnothing l}\right\rangle=\frac{\int d n_{1}^{e q} d n_{2}^{e q} \sigma v_{M \varnothing l}}{\int d n_{1}^{e q} d n_{2}^{e q}}
$$

where

$$
d n_{i}^{e q}=g_{i} \frac{d^{3} p_{i}}{(2 \pi)^{3}} f\left(E_{i}, T\right)
$$

\footnotetext{
${ }^{2}$ We thank F. Feruglio on this point.
} 
$g_{i}$ is the number of internal degrees of freedom for the particle species $i$ and $f\left(E_{i}, T\right)$ is the statistical distribution function for a particle with energy $E_{i}$ in a thermal bath at temperature $T$. The factor $v_{M \varnothing l}=\sqrt{\left(p_{1} \cdot p_{2}\right)^{2}-m_{1}^{2} m_{2}^{2}} /\left(E_{1} E_{2}\right)$ is known as the Møller velocity (see Ref. [12]) and $\sigma$ is the sum over all possible scattering channels of particles 1 and 2.

In general equation (16) can only be solved numerically, but if the particle species $i$ is massless then it is possible to obtain analytic formulae for (16). Since the decoupling temperature of a particle species $i$ will be calculated when $T \gg m_{i}$ it will be a good approximation to use the analytic formulae for massless particles. For a cross section with a power law like behaviour and parametrised as $\sigma=\sum_{i} \hat{\sigma}_{i} s^{n_{i}}$, where $\mathrm{s}$ is the Mandelstam variable one finds that for $\operatorname{Re}\left(n_{i}\right)>-3$

$$
\left\langle\sigma v_{M \varnothing l}\right\rangle=\frac{1}{\zeta(3)^{2}} \sum_{i} \frac{C_{i}}{\left(n_{i}+2\right)}\left[\Gamma\left(n_{i}+3\right) \zeta\left(n_{i}+3\right)\right]^{2} \hat{\sigma}_{i} T^{2 n_{i}}
$$

where $C_{i}=2^{2 n_{i}}-1,\left(\left(2^{2+n_{i}}-1\right)^{2} / 18\right)$ for Bose-Einstein (Fermi-Dirac) statistics, $\zeta$ is the Riemann zeta function and $\Gamma$ is the Euler gamma function. If the cross section has a logarithmic dependence on $s$ then for $\sigma=\hat{\sigma}_{0} \log s+\hat{\sigma}_{1}$ with a boson and fermion in the initial state we obtain

$$
\left\langle\sigma v_{M \varnothing l}\right\rangle=\hat{\sigma}_{0} \log T^{2}+2.63327 \hat{\sigma}_{0}+\hat{\sigma}_{1}
$$

Let us first calculate the annihilation rate for the goldstino. In the limit that $\sqrt{s} \gg m_{3 / 2}, m_{S}, m_{P}$ the dominant goldstino annihilation cross section is that into photons (1). Using the fact that the equilibrium number density of relativisitic goldstinos is $n_{\tilde{G}}=3 \zeta(3) /\left(2 \pi^{2}\right) T^{3}$ the goldstino annihilation rate is [2]

$$
\Gamma_{\tilde{G}}=n_{\tilde{G}}\left\langle\sigma v_{M \varnothing l}\right\rangle \simeq 1.22 \frac{\tilde{M}^{2}}{F^{4}} T^{7} .
$$

Similarly, we can determine the interaction rate for the scalars $S$ and $P$. Comparing the cross section (10) (or (13)) with (12) we find that the scattering of $S, P$ off the background thermal plasma (12) will be the dominant process determining the interaction rate. For the scattering off relativistic fermions with equilibrium number density $n_{f}=3 \zeta(3) / \pi^{2} T^{3}$ the scalar interaction rate becomes

$$
\Gamma_{S f}=n_{f}\left\langle\sigma v_{M \varnothing l}\right\rangle \simeq 0.183 \alpha \frac{\tilde{M}^{2}}{F^{2}} T^{3}\left[\log \left(\frac{T^{2}}{M_{\gamma}^{2}}\right)+0.88\right],
$$

and similarly for $P$. As discussed previously, nonzero cubic scalar couplings can affect the interaction rate at lower temperatures via the s-channel annihilation of scalars into two photons (15). In this scenario with an equilibrium scalar number density $n_{S}=\zeta(3) / \pi^{2} T^{3}$ the interaction rate is given by

$$
\Gamma_{S S}=n_{S}\left\langle\sigma v_{M \varnothing l}\right\rangle \simeq 0.009 A_{S}^{2} \frac{\tilde{M}^{2}}{F^{2}} T .
$$

We will use this rate to make sure that the scalars do not come back into thermal equilibrium during nucleosynthesis. 


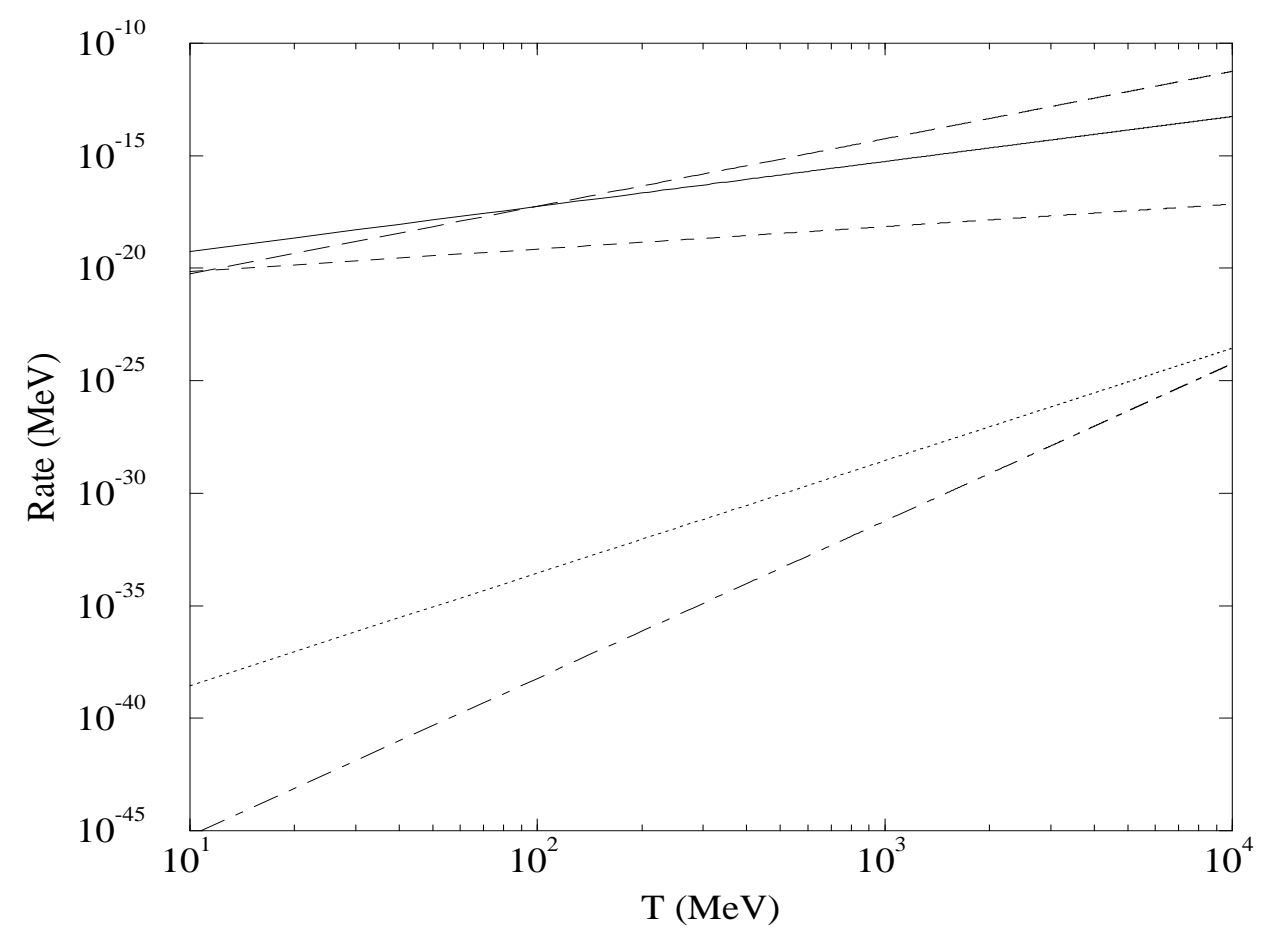

FIG. 1. The Hubble expansion rate (solid line), $\Gamma_{S f}$ (long-dashed line), $\Gamma_{S S}$ s-channel (S exchange) (dashed line), $\Gamma_{S S}$ t-channel ( $\gamma$ exchange) (dotted line) and $\Gamma_{\tilde{G} \tilde{G}}$ (dot-dashed line) as a function of temperature in the early universe. Notice that the goldstino decouples before the spin-0 superpartners.

Notice that for temperatures $T \sim \mathcal{O}(100) \mathrm{MeV}$ the scalar $S$ and $P$ interaction rate (21) is larger than the goldstino rate (20). Consequently $S$ and $P$ will remain in thermal contact with the plasma much longer than the goldstinos. This can be clearly seen when the interaction rates are compared with the Hubble expansion rate of the universe $H$. If we express the energy density of the universe, $\rho(T)$ in terms of the photon energy density $\rho_{\gamma}(T)=\pi^{2} / 15 T^{4}$ then the Hubble expansion becomes

$$
H=\sqrt{\frac{8 \pi G_{N}}{3} \rho}=\frac{\pi}{\sqrt{90}} g_{\rho}^{1 / 2} \frac{T^{2}}{M_{P}},
$$

where $g_{\rho}$ is the effective number of relativisitic degrees of freedom. In Figure 1 we have shown how the various interaction rates compare with the Hubble expansion rate for the representative values of $g_{\rho}=65 / 4, \tilde{M}=100 \mathrm{GeV}, F=3.8 \times 10^{9} \mathrm{GeV}^{2}$ and we have used $M_{\gamma} \sim \sqrt{\alpha} T$. It is clear from the figure that the goldstino will decouple at times much earlier than the spin-0 scalar superpartners and that the dominant scattering processes for $S$ and $P$ come from the scattering off background fermions (21). Also in the figure we can see the linear temperature dependence of (22) (with $A_{S}=0.01 \mathrm{GeV}$ ) and the possibility of $\Gamma \gtrsim H$ at lower temperatures.

Let us now obtain the expressions for the decoupling temperature. In principle to determine the decoupling temperatures of $S, P$ and $\tilde{G}$ we would need to solve a set of coupled 
Boltzmann equations. However, in practice since the interaction rates are significantly different between the scalar superpartners and the goldstino one finds that the coupled differential equations become separate equations controlled by ratios of $\Gamma / H$. In particular the Boltzmann equations for $S(P)$ separate when the dominant interaction rate is $\Gamma_{S f}\left(\Gamma_{P f}\right)$ since the equations do not involve either of the other species. Thus it will be sufficient for our purposes to consider the equation $\Gamma \sim H$ for the dominant interaction rate $\Gamma$. When the cubic scalar couplings are included we will also compare the interaction rate with $H$ even though the equations may become coupled. Again this will be sufficient in order to obtain an order of magnitude estimate.

When the cross section has power law behaviour the decoupling temperature may be easily obtained analytically. One finds for the goldstino decoupling temperature the result

$$
T_{\tilde{G}} \simeq 0.77 g_{\rho}^{1 / 10}\left(\frac{F^{4}}{\tilde{M}^{2} M_{P}}\right)^{1 / 5} .
$$

For the spin-0 superpartners the dominant scattering mode is $\Gamma_{S f}$. In this case, an analytic expression for the decoupling temperature of the spin-0 superpartners of the goldstino can be obtained since for a plasmon mass $M_{\gamma} \sim \sqrt{\alpha} T$ the temperature dependence in the logarithmn cancels in $\Gamma_{S f}$. Thus by requiring $\Gamma_{S f} \sim H$ the decoupling temperature is given by

$$
T_{S} \simeq \frac{1.81}{(0.88-\log \alpha)} \frac{g_{\rho}^{1 / 2}}{\alpha}\left(\frac{F^{2}}{\tilde{M}^{2} M_{P}}\right)
$$

Since in limit $\sqrt{s} \gg m_{3 / 2}, m_{S}, m_{P}$ the scalars $S, P$ decouple later than the goldstino (see also the figure) we will use (25) to set a lower bound on the scale of supersymmetry breaking $F$. Note that, without loss of generality we are also implicitly assuming that $S$ and $P$ decouple at the same time.

When the cubic scalar couplings are included the cross section (15) is again a power law and the analytical expression for the decoupling temperature is

$$
T \simeq 0.03 g_{\rho}^{-1 / 2} A_{S}^{2}\left(\frac{\tilde{M}^{2} M_{P}}{F^{2}}\right) .
$$

We will need to make this temperature as low as possible in order that the fields $S$ (and $P$ ) do not come back into thermal equilibrium during nucleosynthesis.

It is also instructive to consider the limit $m_{S}, m_{P} \gg \sqrt{s} \gg m_{3 / 2}$ in which only the goldstino is assumed to be in thermal contact with the heat bath at temperatures $T \sim$ $\mathcal{O}(\mathrm{MeV})$. As noted earlier, in this limit the goldstino annihilation cross section into photons changes to the form (2). Now the annihilation cross section into photons and fermions are comparable and qualitatively similar $\sigma \propto s^{3}$. Including the electron, muon and 3 families of massless neutrinos for the fermions gives for the decoupling temperature

$$
T_{\tilde{G}} \simeq 0.53 g_{\rho}^{1 / 14}\left(\frac{F^{4}}{M_{P}}\right)^{1 / 7}
$$


This equation will be used to show how much weaker the bound on the supersymmetry breaking scale $F$, becomes in the massive $m_{S}, m_{P}$ case.

In order to bound the decoupling temperature of a particle species $i$, we need to consider its effect on nucleosynthesis. This can be done by noting that the energy density of new massless particles is equivalent to an effective number $\Delta N_{\nu}$ of additional doublet neutrinos

$$
\Delta N_{\nu}=f_{B, F} \sum_{i} \frac{g_{i}}{2}\left[\frac{g_{\rho}\left(T_{\nu}\right)}{g_{\rho}\left(T_{D_{i}}\right)}\right]^{4 / 3},
$$

where $f_{B}=8 / 7$ for bosons, $f_{F}=1$ for fermions and $g_{i}$ is the number of internal degrees of freedom of the particle species $i$ [13]. If the scalars decouple at $T_{S, P}=T_{\nu} \simeq \mathcal{O}(1 \mathrm{MeV})$ then according to (28) $S$ and $P$ will effectively behave as 1.14 extra neutrino doublets. If, however the scalars were to decouple during the epoch $T_{\nu}<T_{S, P}<T_{\mu}$, where $T_{\mu}$ is the muon decoupling temperature then the effective number of additional doublet neutrinos during nucleosynthesis due to $S$ and $P$ is 0.91 . While neither of these bounds can be strictly ruled out [14] we will nevertheless suppose that $S$ and $P$ decouple before the muon decouples 3 to quote a bound on the supersymmetry breaking scale $F$.

As alluded to earlier there is no loss of generality if we calculate the bounds using the expressions for the scalar field $S$. The bounds are identical if we were to use the expressions for $P$, since to leading order the dominant cross sections have no dependence on $m_{S}$ or $m_{P}$. Thus using the expression (25) and requiring that $T_{S}>T_{\mu} \simeq \mathcal{O}(100 \mathrm{MeV})$ leads to the bound

$$
F \gtrsim 4 \times 10^{9} \mathrm{GeV}^{2}\left(\frac{\tilde{M}}{100 \mathrm{GeV}}\right) .
$$

This strengthens the bound considered in [2], where the effects of massless $S$ and $P$ during nucleosynthesis were not considered. For $M \simeq 100 \mathrm{GeV}$ the bound on the supersymmetry breaking scale becomes $F \gtrsim 4 \times 10^{9} \mathrm{GeV}^{2}$. Using the relation $F=\sqrt{3} m_{3 / 2} M_{p}$ this leads to a bound on the gravitino mass $m_{3 / 2} \gtrsim 1 \mathrm{eV}$. This is similar to the bound obtained by [6] where the logarithmic term was neglected. One may have expected a large enhancement from the logarithmn factor in the massless limit due to the singularity, but since the plasmon mass is large, the enhancement in the cross section is only $\log \alpha \sim-5$ and thus impotent. Also if the mass of the scalars $m_{S, P} \gtrsim 1 \mathrm{MeV}$ then the bounds calculated here would be void because the cross section limits are no longer valid and a more detailed analysis in this intermediate mass range would need to be done.

When the cubic scalar couplings, represented by $A_{S}$ are included we need to make sure that they do not return to thermal equilibrium during nucleosynthesis. Requiring $T \lesssim \mathcal{O}(\mathrm{eV})$ leads to a bound of $m_{3 / 2} \gtrsim 10^{-4} A_{S}$, which vanishes in the limit $A_{S}=0$. We emphasise that while this bound is approximate, a more detailed analysis than that given here could provide interesting constraints on theories with nonzero cubic scalar couplings.

3 This is somewhat plausible given that there may be other contributions to $\Delta N_{\nu}$ arising from massive neutrinos. 
Finally, the gravitino mass bound coming from (27) in the limit $m_{S}, m_{P} \gg \sqrt{s} \gg m_{3 / 2}$ is $m_{3 / 2} \gtrsim 4 \times 10^{-7} \mathrm{eV}$ and is independent of the gaugino mass $\tilde{M}$. As expected the bound becomes much weaker in this case and there are already stronger bounds from collider phenomenology in this limit [9].

\section{CONCLUSION}

We have shown that in the massless limit the scalar superpartners $S, P$ of the goldstino couple much more strongly to the background thermal plasma than the goldstino. This means that during nucleosynthesis the scalars $S$ and $P$ may contribute as extra effective neutrino doublets, while the goldstinos have long since decoupled. Requiring that $S$ and $P$ decouple sufficiently early, so as not to upset current nucleosynthesis bounds, sets a lower bound on the scale of supersymmetry breaking which depends on the gaugino mass $\tilde{M}$. The bound on the supersymmetry breaking scale assuming $\tilde{M}=100 \mathrm{GeV}$ is $\sqrt{F} \gtrsim 60 \mathrm{TeV}$ which leads to a lower bound on the gravitino mass of $m_{3 / 2} \gtrsim 1 \mathrm{eV}$. These bounds are much stronger than those considered in [2], but complement the cosmological bounds in [6]. Finally in the massive $S, P$ case the nucleosynthesis bound is considerably weaker and similar to other recent cosmological bounds [15, [6].

\section{ACKNOWLEDGEMENTS}

We would like to thank F. Feruglio, G. Jungman and A. Riotto for useful discussions. 


\section{REFERENCES}

[1] For a recent review, see: S.P. Martin, hep-ph/9709356.

[2] T. Gherghetta, Nucl. Phys. B485, 25 (1997).

[3] A. Brignole, F. Feruglio and F. Zwirner, Nucl. Phys. B501, 332 (1997).

[4] A. Brignole, F. Feruglio and F. Zwirner, hep-th/9709111; F. Feruglio, hep-ph/9711270.

[5] M.A. Luty and E. Ponton, hep-ph/9706268.

[6] J.A. Grifols, R.N. Mohapatra and A. Riotto, Phys. Lett. B400, 124 (1997); Phys. Lett. B401, 283 (1997).

[7] T. Bhattacharya and P. Roy, Phys. Lett. B206, 655 (1988); Nucl. Phys. B328, 481 (1989).

[8] T. Bhattacharya and P. Roy, Phys. Rev. D38, 2284 (1988).

[9] A. Brignole, F. Feruglio and F. Zwirner, hep-ph/9711516.

[10] M. Nowakowski and S.D. Rindani, Phys. Lett. B348, 117 (1995).

[11] M. Joyce, T. Prokopec and N. Turok, Phys. Rev. D53, 2930 (1996).

[12] P. Gondolo and G. Gelmini, Nucl. Phys. B360, 145 (1991).

[13] S. Sarkar, Rept. Prog. Phys. 59, 1493 (1996), hep-ph/9602260.

[14] S. Sarkar, hep-ph/9710271.

[15] J.A. Grifols, E. Masso and R. Toldra, hep-ph/9707536.

[16] D.A. Dicus, R.N. Mohapatra and V.L. Teplitz, hep-ph/9708369. 